\title{
The Saturnian satellite Rhea as seen by Cassini VIMS
}

Katrin Stephan (1), Ralf Jaumann (1,2), Roland Wagner (1), Roger Clark (3), Dale P. Cruikshank (4), C.A. Hibbitts (5), Thomas Roatsch (1), R. H. Brown (6), Bonnie J. Buratti (7), G. Filacchione (8), F. Cappacione (8), Kevin H. Baines (7), Phil D. Nicholson (9). (Katrin.Stephan@dlr.de / Fax :+493067055 402).

(1) Institute of Planetary Research., Berlin, Germany; (2) FU Berlin, Germany; (3) U.S. Geological Survey, Denver, USA; (4) NASA Ames Res. Center, Mountain View, USA; (5) APL, Laurel, USA, (6) LPL, University of Arizona, Tucson, USA (7) JPL, Pasadena, USA; (8) INAF-IASF, Roma, Italy; (9) Cornell University, Ithaca, USA.

\section{Introduction}

The icy Saturnian satellite Rhea is often regarded as twin to its neighboring satellite Dione especially with respect to its geological history $[1,2]$ and its spectral surface properties [3]. Cassini VIMS detected the satellites surfaces in the wavelength range from 0.35 to $5.1 \mu \mathrm{m}$ and offers the first spatially resolved hyperspectral data of the Saturnian satellites [4], which allow a detailed comparison of the spatial distribution of the spectral properties of Rhea in comparison the results achieved for Dione $[5,6]$.

\section{VIMS observations}

VIMS data acquired during 24 flybys (up to Cassini's $54^{\text {th }}$ orbit in Dec. 2007) exhibiting pixel ground resolutions of at least $100 \mathrm{~km}$ per pixel were incorporated into this study, analyzed, map-projected and combined into VIMS mosaics [6,7]. Whereas most observations exhibit relatively low pixel ground resolutions of less than $50 \mathrm{~km}$ per pixel VIMS observations of Rhea's anti-Saturnian hemisphere acquired during one non-targeted flyby (Cassini orbit 49 on Aug. 30, 2007) reach pixel ground resolutions up to $1 \mathrm{~km}$ per pixel. In order to relate the spectral variations to geological and morphological surface features the derived VIMS maps were overlaid onto base maps derived from Voyager ISS and Cassini ISS cameras at visible wavelengths.

\section{Major geological units:}

Voyager images showed that Rhea exhibits a similar distribution of geological units like its inner neighbor Dione [1,2] with densely cratered plains dominating Rhea's leading hemisphere with average cratering model ages of about $4.1 \mathrm{Gyr}[8,9]$ or 3.6 Gyr [10] and bright, filament-like wispy markings characterizing the trailing hemisphere verified as tectonic graben systems by Cassini ISS data [8]. Abundant old large craters and multiring impact basins, though heavily degraded, were detected in a digital elevation model (DEM) derived by ISS data [8]. Rhea's most prominent ray crater Inktomi $\left(12.5^{\circ} \mathrm{S} / 112^{\circ} \mathrm{W}\right)$ with crater model ages of $280 \mathrm{Myr}$ or 8 Myr as derived by [7], using chronology models by [8] (lunar-like cratering rate, higher age) and [9] (constant cratering rate, lower age) probably represents the youngest surface feature on Rhea.

\section{Spectral variations}

Although Rhea's surface is mainly composed of water ice [10] distinct spatial variations of its spectral properties could be derived (Fig. 1) that appear to be similar to the neighboring satellite Dione [2,3]. In order to avoid influences of the viewing geometries (i.e. phase angle) only band depth measurements of water ice absorptions were combined into the resulting maps. Fig. 1 shows the variations in band depth of the water ice absorption at $1.5 \mu \mathrm{m}$ indicating varying amount, sizes of the particles, and/or crystallinity of water ice. In general, water ice absorptions are distinctly deeper than on Dione (Fig. 2) but still slightly weaker than on Enceladus.

No indications of polar caps (mostly due to inclomplete coverage) or endogenic activity that could be related to any contribution to the E-ring material or impacting of E-ring particles preferably onto Rhea's leading hemisphere could be clearly identified. As observed on Dione, clean ice deposits on the leading hemisphere extend from a geologically young impact crater named Inktomi. Its ejecta representing excavated material imply more or less clean ice in Rhea's crust. Similar to the ray crater Creusa on Dione [6] Inktomi exhibits the largest ice particles measured on this satellite's surface. Its extended ejecta dominate most parts of the leading hemisphere, which has a strong effect onto global 
hemispherical spectral differences (Fig. 2).

Instead, icy regions on the trailing side occur in the vicinity of the prominent tectonic graben systems. The remaining parts of this hemisphere are characterized by a concentration of non-ice material. Although, the variations are less pronounced than measured on Dione (Fig. 2) their spatial distribution nevertheless indicate a similar mechanism responsible for them i.e. magnetospheric particles that impact onto the trailing hemisphere. This is also supported by similar major spectral characteristics of the dark material on Rhea and Dione (Fig. 2) [5]. The lesser but still distinct degree of contamination is probably related to different positions of both satellites within Saturn's magnetosphere.

\section{References}

[1] Smith et al. (1981), Science, 212, 163-191; [2] Plescia (1983) Icarus 56, 255-277 ; [3] Clark R. N. et al. (1986), in Saturn, UofA Press, Tucson, Az., p. 437-491; [4] Brown, R.H. et al. (2005) SSR, 115, 115-18; [5] Clark et al. (2008) Icarus, 193, 372-386 ; [6] Stephan et al. (2009) Icarus; [7] Jaumann et al., 2006 PSS ; [8] Wagner et al. (2007) LPSC XXXVIII [9] Neukum [10] Zahnle [11] Wagner et al. (2006), LPSC XXXVII, 1805; [12] Stephan et al. (2008a), LPSC XXXIX, 1717.

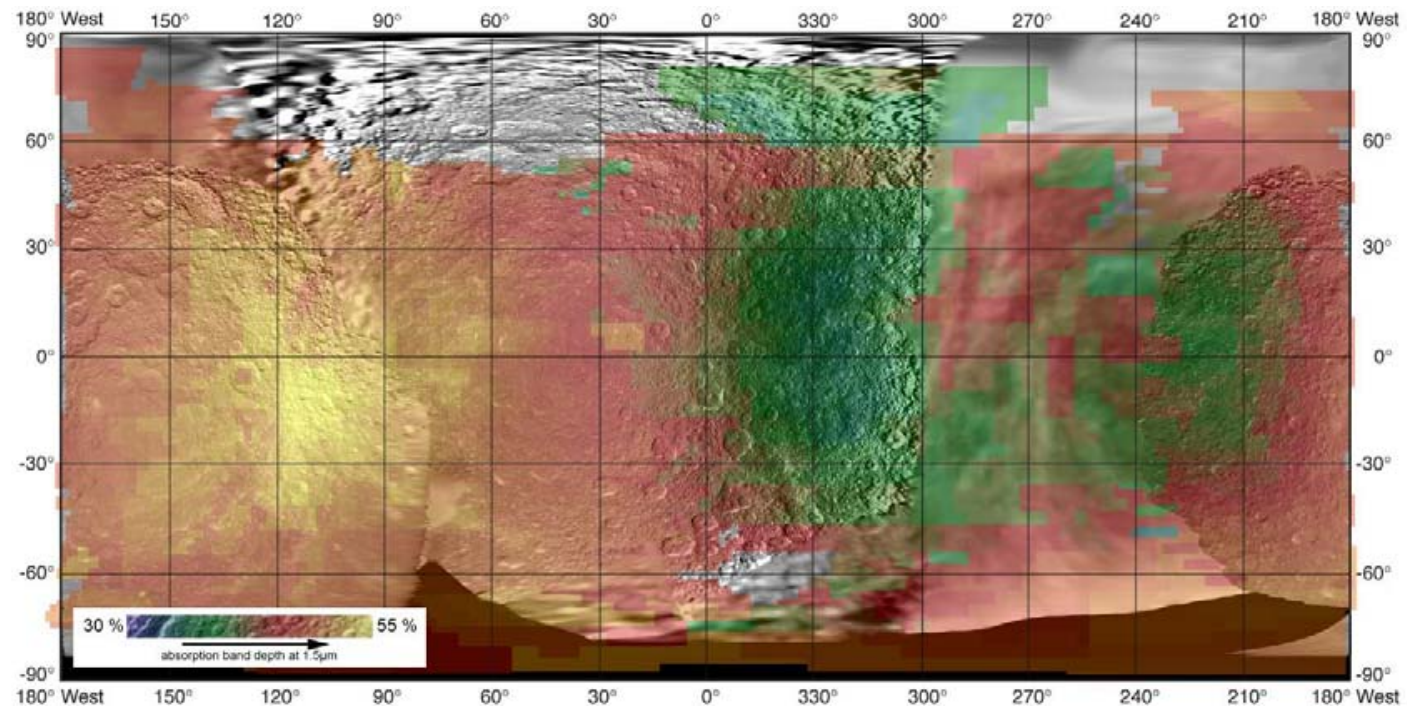

Fig. 1: Global VIMS map showing the band depth variations of the ice absorption at $1.5 \mu \mathrm{m}$ (increasing depth as an indicator for an increasing spectral dominance of water ice) overlaid onto a Voyager/Cassini ISS basemap.
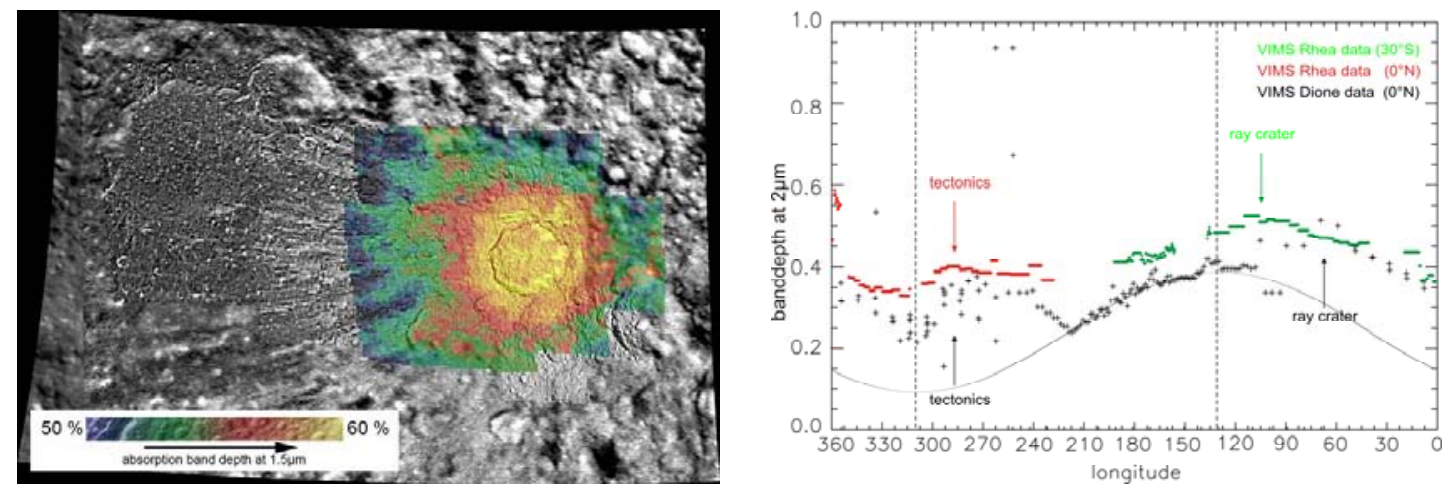

Fig. 2: High-resolution VIMS data of the ray crater Inktomi $\left(30^{\circ} \mathrm{N}, 0^{\circ} \mathrm{N}\right.$, and $\left.30^{\circ} \mathrm{S}\right)$ overlaid onto simultaneously acquired Cassini ISS images (left). Comparison of spectral variations along specific latitudes between Rhea and Dione (right). 